\title{
Alglucosidase alfa enzyme replacement therapy as a therapeutic approach for a patient presenting with a PRKAG2 mutation
}

\author{
Stephanie L. Austin a , Andrew Chiou ${ }^{a}$, Baodong Sun ${ }^{\text {a }}$, Laura E. Case ${ }^{\text {b }}$, Kenny Govendrageloo ${ }^{c}$, \\ Perrin Hansen ${ }^{\mathrm{d}}$, Priya S. Kishnani ${ }^{\mathrm{a}, *}$ \\ a Division of Medical Genetics, Department of Pediatrics, Duke University Medical Center, Durham, NC, USA \\ ${ }^{\mathrm{b}}$ Doctor of Physical Therapy Division, Duke University Medical Center, Durham, NC, USA \\ c Pediatric Cardiology, Netcare Sunninghill Hospital, Johannesburg, South Africa \\ ' Pediatric Neurology, Netcare Waterfall City Hospital, Johannesburg, South Africa
}

\section{A R T I C L E I N F O}

\section{Article history:}

Received 26 September 2016

Accepted 26 September 2016

Available online 28 September 2016

\section{Keywords:}

Glycogen storage disease

Cardiomyopathy

AMP-activated protein kinase

Acid alpha-glucosidase

PRKAG2

\begin{abstract}
A B S T R A C T
Objective: PRKAG2 syndrome, an autosomal dominant disorder, is characterized by severe infantile hypertrophic cardiomyopathy and heart rhythm disturbances to cases with a later presentation and a spectrum of manifestations including cardiac manifestations, myopathy and seizures. The cardiac features of PRKAG2 resemble the cardiac manifestations of Pompe disease. We present a patient who was initially diagnosed with Pompe disease and treated with alglucosidase-alfa enzyme replacement therapy (ERT); however, he was eventually diagnosed to carrying a PRKAG2 pathogenic gene mutation; he did not have Pompe disease instead he was a carrier for the common adult leaky splice site mutation in the GAA gene.

Case report: At 2.5 months, the patient had hypotonia/generalized muscle weakness, a diagnosis of non-classic infantile Pompe disease was made based on low acid alpha-glucosidase activity and the patient started on ERT at 11 months. However, 1 month later, the patient began to have seizures. As the patient's medical history was somewhat unusual for infantile Pompe disease, further evaluation was initiated and included a glycogen storage disease sequencing panel which showed that the patient had a pathogenic mutation in PRKAG2 which had been reported previously. ERT was discontinued and patient had a progression of motor deficits. ERT was reinitiated by the treating physician, and a clinical benefit was noted.

Conclusion: This report outlines the benefits of ERT with alglucosidase alfa in a patient with PRKAG2 syndrome, the decline in his condition when the ERT infusions were discontinued, and the significant positive response when ERT was reinitiated.
\end{abstract}

(c) 2016 Elsevier Inc. All rights reserved.

\section{Introduction}

In humans, glycogen is a polymer of glucose that is stored in liver, skeletal muscle, and heart cells. There are a number of enzymes involved in the synthesis and breakdown of glycogen within the body. Deficiency or dysfunction of one of these enzymes results in a group of diseases called glycogen storage diseases (GSDs), in which the clinical hallmark is excessive glycogen accumulation in various tissues [1].

Abbreviations: ERT, enzyme replacement therapy; GAA, acid alpha-glucosidase; GSD, glycogen storage diseases; AMPK, AMP-activated protein kinase; rhGAA, recombinant human GAA; IVS, interventricular septum; LVPW, left ventricular posterior wall; PT, physical therapy; AST, aspartate aminotransferase; ALT, alanine transaminase; MABC, Movement ABC; MDC, minimal detectable change; MID, minimal important difference; M-FUN, Miller Function and Participation Scales; GLUT4, glucose transporter 4; G6P, glucose 6-phosphate; GS, glycogen synthase; PAS, periodic acid-Schiff.

* Corresponding author at: Medical Genetics, Duke University Medical Center, Box 103856 DUMC, Durham, NC 27710, USA.

E-mail address: priya.kishnani@dm.duke.edu (P.S. Kishnani).
One such GSD is PRKAG2 syndrome, which is caused by mutations in the PRKAG2 gene that encodes the $\gamma 2$ subunit of AMP-activated protein kinase (AMPK). AMPK is a crucial cellular energy sensor that regulates a number of vital cellular metabolic cascades and lipid/glucose metabolic pathways [2-4]. PRKAG2 syndrome is an autosomal dominant disorder with a wide spectrum of symptoms. The disease phenotype is a spectrum that is characterized by severe infantile hypertrophic cardiomyopathy and heart rhythm disturbances to cases with a later presentation (age range 8 to 42 years of age) of cardiac manifestations such as increased left ventricular wall thickness and ventricular pre-excitation [5]. Also, symptoms of myalgia, myopathy, and seizures have been reported further expanding the phenotype and characterization of it beyond a cardiac syndrome as was previously thought [6,7]. Mutations in the PRKAG2 gene cause chronic activation of AMPK and the underlying mechanism of excess glycogen accumulation in PRKAG2 syndrome is illustrated in Fig. 1 [2,8,9]. The clinical cardiac features and muscle weakness of PRKAG2 syndrome closely resemble the cardiac and muscle manifestations of Pompe Disease (GSD Type II). Pompe disease is 


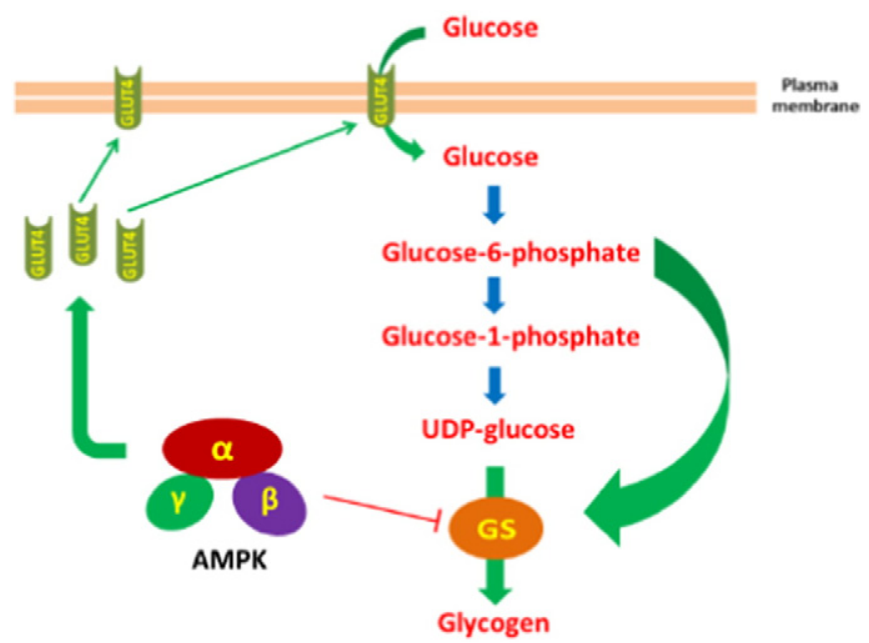

Fig. 1. Schematic mechanism of AMPK-mediated increase in cardiac and skeletal muscle glycogen accumulation in PRKAG2 deficiency (adapted from Hunter et al. [9] \& Moffat and Harper [2]). Mutations in the PRKAG2 gene, which encodes the regulatory $\gamma 2$ subunit, cause chronic activation of AMPK. Elevated AMPK activity promotes glucose transporter 4 (GLUT4) shuttling to the plasma membrane and increases glucose uptake and intracellular glucose 6-phosphate (G6P) concentration. This leads to an allosteric activation of glycogen synthase (GS), which overrides the inhibitory effect of AMPK on $\mathrm{GS}$, resulting in a net increase in GS activity and excess glycogen storage in muscle cells.

an autosomal recessive metabolic disorder that is characterized by glycogen accumulation in lysosomes of cardiac, skeletal, and smooth muscles due to the deficiency of the lysosomal enzyme acid alphaglucosidase (GAA) [10]. Enzyme replacement therapy (ERT) with recombinant human GAA (rhGAA, Alglucosidase alfa, Genzyme, Boston, MA, USA) is currently the only FDA approved treatment for Pompe disease.

With the phenotypic similarity of PRKAG2 syndrome to Pompe disease, there is the potential for a misdiagnosis for either of these disorders, especially the infantile form of Pompe disease. Here, we present an interesting case of a patient initially diagnosed with Pompe disease and started on ERT with alglucosidase alfa, which improved his condition. However, over the course of the therapy, the patient began to develop inconsistent symptoms that led his physicians to question the diagnosis. Through further medical tests, he was diagnosed with PRKAG2, carrying a pathogenic mutation in the gene, and being a carrier of Pompe disease and not affected. This case report outlines the benefits that the patient showed while on ERT treatment, the decline to his condition when his infusions were discontinued due to his updated diagnosis, and the significant positive response when alglucosidase alfa ERT was reinitiated. This case study provides several key messages: 1) the importance of confirming the diagnosis of Pompe disease via gene sequencing and doing enzyme analysis in a lab with experience before ERT initiation, 2) our group's previous work showing the potential of GAA as a treatment approach for cytoplasmic GSDs [11] such as PRKAG2, and 3) it also supports the expanded phenotype of PRKAG2 depicting a case with myopathy, seizures, and minimal cardiac involvement.

\section{Case presentation}

This male patient was born by caesarian section at 38 weeks gestation as a result of the nuchal cord being wrapped around his neck. At age $21 / 2$ months, the patient was noted to have hypotonia and generalized muscle weakness. He was areflexic and had feeding difficulties. At age 4 months, the patient began developing severe lower respiratory infections which led to frequent admissions to the hospital. Labs at 11 months of age showed a mild increase in creatine kinase (CK) at $197 \mathrm{IU} / \mathrm{L}$ (normal range: 38-174 IU/L) and creatine kinase MB (CKMB) at $8.3 \mathrm{ng} / \mathrm{mL}$ (normal range: $0.8-5.1 \mathrm{ng} / \mathrm{mL}$ ) while other labs including alanine transaminase (ALT) (15 IU/L; normal range: $<45 \mathrm{IU} / \mathrm{L}$ ) and aspartate aminotransferase (AST) (47 IU/L; normal range: 9-80 IU/L) were normal. Following numerous recurrent pneumonias, and the history of muscle weakness, the patient's physicians raised Pompe disease as a potential diagnosis. Blood GAA enzyme testing done locally revealed a deficiency (4.81 units versus 18.66 units in the control sample). An ECHO revealed mild hypertrophy of the interventricular septum (IVS) and a normal sized left ventricular posterior wall (LVPW) with a normal left ventricular mass. The ECG showed that the ventricular forces were normal with a SR of 146/min and a PR interval of 0.10 . Given the early findings of hypotonia, mild hypertrophy of IVS, and the low GAA enzyme activity, a diagnosis of non-classic infantile Pompe disease was made. The patient was initiated on ERT with alglucosidase alfa at a dose of $20 \mathrm{mg} / \mathrm{kg}$ every 2 weeks at age 11 months.

At age 11 months, the patient was belly crawling asymmetrically and was unable to achieve sitting from a prone or supine position. Due to weakness in his neck and trunk flexors, the patient consistently sat with his trunk collapsed in a kyphotic position and his head propped up in capital extension in a chin poke position. This weakness also caused the patient to struggle with clearing secretions while coughing. Three months after the initiation of ERT, the patient's gross motor abilities as assessed by his physical therapist began improving and he achieved new milestones. He was able to crawl more efficiently, to achieve sitting independently from a prone or supine position, and to pull himself up into a standing position without aid. The patient's level of endurance also improved which allowed him to be more active. At age 29 months, the patient was able to walk independently with an age appropriate gait pattern and to climb small steps as well as jump off the steps without support. He was able to transition independently into and out of any position, which helped him participate more fully in activities appropriate for his age.

Interestingly at age 13 months, 1 month after the start of ERT, the patient began to have febrile and non-febrile seizures. An EEG completed at age 14 months revealed epileptic activity. He exhibited tremors of the head and extremities at intermittent intervals with the tremors growing worse upon awakening and while in the motion of reaching. The patient was evaluated by a neurologist $(\mathrm{PH})$ and was noted to have an intention tremor, titubation, ataxia, and very mild hypotonia. At age 4 years, he developed complex partial seizures compounded by a 2 to 3 day period where he was completely floppy and weak, often unable to lift his head off the pillow. Based on these events, the neurologist diagnosed the patient with a complex genetic epilepsy syndrome. As the patient's medical history was somewhat unusual for infantile Pompe disease, further evaluation was initiated to determine if he had another diagnosis in addition to Pompe disease to explain these findings or if the initial diagnosis was incorrect. At about age 2.5 years (30 months), GAA enzyme activity performed at the Duke Biochemical Genetics lab on skin fibroblasts was suggestive of a carrier status (50.7 $\mathrm{nmol} / \mathrm{h} / \mathrm{mg}$ with a control range of $45-180 \mathrm{nmol} / \mathrm{h} / \mathrm{mg}$ and an affected range of $0-20 \mathrm{nmol} / \mathrm{h} / \mathrm{mg}$ ). Sequencing of the GAA gene found the patient to be heterozygous for the common splice site mutation c.-32-13 $\mathrm{T} / \mathrm{G}$, a pathogenic mutation among patients with late onset Pompe disease. No other mutation was identified; these findings were consistent with carrier status of Pompe disease. ERT was discontinued for the patient at age 33 months after 22 months on ERT. A quadriceps muscle biopsy obtained at age 44 months showed cytoplasmic glycogen, suggestive of a non-lysosomal glycogen storage disease (Figs. 2 and 3). Muscle acid alpha glucosidase activity tested in the low normal range suggestive of carrier status; phosphorylase and phosphorylase kinase activities measured in normal ranges. Further work up included a mitochondrial myopathy enzyme panel and a mitochondrial respiratory chain panel which were normal and a glycogen storage disease sequencing panel (GCTS Pathology, London, UK) which showed that the patient had a pathogenic mutation in PRKAG2, c.298G > A (Gly100Ser/ G100S), which had been reported previously in cases of PRKAG2 (Table 1). As PRKAG2 is inherited in an autosomal dominant manner, 


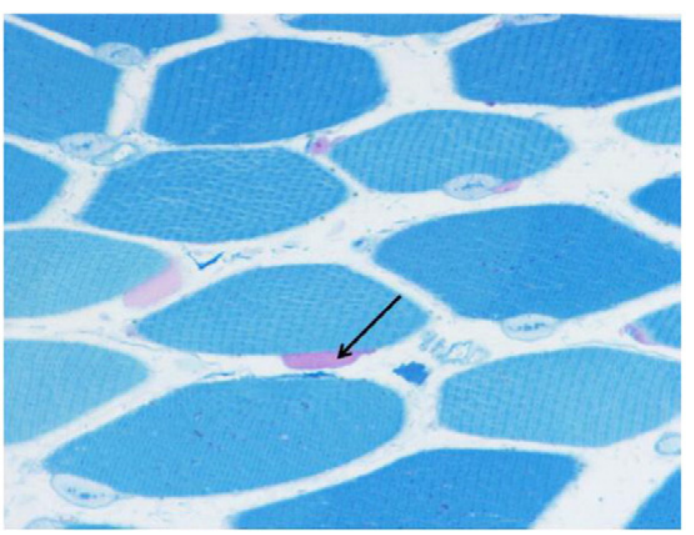

Fig. 2. High resolution light microscopy of quadriceps muscle biopsy at age 44 months. Patient was not on ERT at the time of biopsy (off ERT for 11 months). One-micron semithin epon sections were stained with Richardsons/periodic acid-Schiff (PAS) stain combination. PAS positive blebs (arrow), are present at the periphery of some cells, suggestive of glycogen accumulation.

the family history was taken again with a focus on the patient's maternal male cousin once removed who suddenly died at age 29 due to a cardiac event. The hospital records indicated that the EKG taken at the day of his death displayed a normal sinus rhythm with normal repolarization, normal PR and QTc with no Brugada pattern. There was mild ST segment depression in the inferior leads.

During the period of 14 months without ERT (age 33 months to age 47 months), the patient was followed closely and clinical decline was noted. The physical therapist observed that the patient was struggling to participate in physical therapy (PT) sessions, which had been easily managed previously while on ERT. He was falling frequently. The patient, who had previously been very interactive during sessions, tended to lie on the floor or on the equipment with significant lack of energy. In addition, there was a decline in his speech and communication; the patient often mumbled and refused to answer his therapist when prompted.

Due to the patient's regressions, past clinical benefit, family request, support from his physicians, and past literature revealing a potential role of alglucosidase alpha in individuals with a cytoplasmic GSD [14], the patient received IV alglucosidase alfa treatment for 6 months on a trial basis with close follow up after the reinitiation of therapy (started at age 47 months). Baseline assessments were done which included tests for AST (44 IU/L; normal range: $<45 \mathrm{IU} / \mathrm{L}$ ), ALT (20 IU/L; normal range: 9-80 IU/L), along with PT measures looking at muscle strength and function measures. After 5 months of ERT, the patient showed

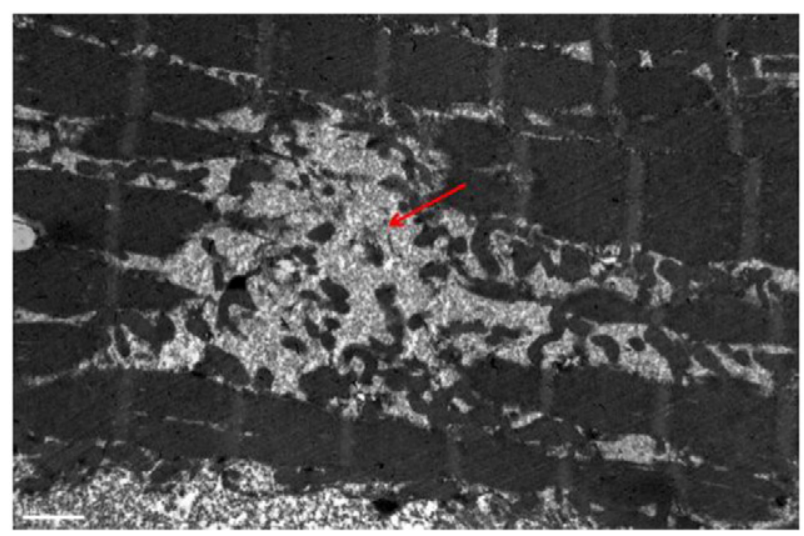

Fig. 3. Electron microscopy of quadriceps muscle biopsy at age 44 months. Patient was not on ERT at the time of biopsy (off ERT for 11 months). The myofibrillar structure of the myocytes was largely intact in most fields. There were isolated foci of frayed and degenerated myofibrils interrupted by small pools of cytoplasmic glycogen. significant improvement according to his neurological, occupational therapy, and physical therapy reports. The patient no longer exhibited myopathic faces or ptosis, his tenting of his upper lip had improved, and he had more facial expression than before. Upon examination, the patient has developed more defined calf muscles, along with an improvement of his strength and power. According to his neurologist $(\mathrm{PH})$, in addition to improvement in strength, the patient's seizures appear to be better controlled since the reinitiation of ERT with alglucosidase alfa; however, this is difficult to understand given that ERT does not cross the blood brain barrier. He had grown physically stronger and did not have episodes in which he lacked energy for consecutive days, become completely floppy, and was unable to hold his head up properly following a seizure.

His PT reports indicated that he was learning new motor skills or improving his current skills to the average level for motor tasks. The patient's physical therapy reports from before ERT reinitiation and post-5 months have recorded significant improvements based on assessment with Movement ABC (MABC) [12] which provides quantitative and qualitative data about a child's performance of ageappropriate tasks within 3 subsections: 1) Manual Dexterity, 2) Ball Skills, and 3) Static and Dynamic Balance. At baseline, according to the MABC assessment, the patient achieved a Total Test Score of 68 ranking him in the 25th percentile. Five months after reinitiation of ERT, the patient achieved a Total Test Score of 73 on his MABC assessment, ranking him in the 37th percentile. A minimal detectable change (MDC) for the $\mathrm{MABC}$ has been reported as 1.21 points, representing a true change in motor function [13]. The results in this child show an increase of 5 points over 5 months, which is greater than the minimal important difference (MID) of the MABC, which has been reported as a change of 2.5 points shown over 6 months [13]. According to his occupational therapist, the patient presented with less fatigue on reassessment and was able to complete the full assessment, which he had initially been unable to accomplish. After 5 months of ERT, he also demonstrated an improvement in his visual motor, fine motor, and gross motor skills as measured by the Miller Function and Participation Scales (M-FUN) [14] when compared to his initial assessment scores taken in his first month of ERT reinitiation. The overall assessment findings at 5 months indicated that he presented with less fatigue and was able to complete the full assessment which he was not able to on initial assessment.

These findings led the patient's physicians to recommend the continuation of the infusions with rhGAA. The patient continues on ERT at the age of 7 years (about 2 years on ERT). Per parental report, he continues to have gross motor gains but some fine motor fatigue with hand writing for longer periods of time and hypotonia. An echocardiogram at 6 years ( 1 year 2 months of ERT) showed no obvious septal defects. Cardiac testing and exam noted a normal heart structurally and functionally with no evidence of pre-excitation or hypertrophic cardiomyopathy.

\section{Discussion}

Due to similar symptomatic phenotypes, rare PRKAG2 cases can be misdiagnosed with infantile Pompe disease. PRKAG2 should be in the differential diagnosis of cases with cardiomyopathy. Interestingly, the patient in our case study only exhibited mild cardiac hypertrophy. There is variability of cardiac involvement in PRKAG2 as shown in Table 1 . He did have a family member die of a sudden cardiac event, and based on current literature, there is a broader clinical spectrum of this disorder beyond cardiac involvement which includes myalgia, myopathy and seizures [6,7]. This patient was diagnosed with a pathogenic mutation in PRKAG2, Gly100Ser. Other patients exhibiting the same PRKAG2 Gly100Ser mutation have been reported to have a variable cardiac presentation including ventricular preexcitation, progressive conduction system disease and ventricular hypertrophy [15]. The family in the Zhang et al. paper did not have muscle symptoms or seizures reported as documented in our patient. However, in one study of 45 cases 
Table 1

Literature review of PRKAG2 cases with symptoms and genotype.

\begin{tabular}{|c|c|c|c|c|c|}
\hline Reference & $\begin{array}{l}\text { Number } \\
\text { of cases }\end{array}$ & Ages & Resolution & Symptoms shown & PRKAG2 genotype \\
\hline Zhang et al. (2013) & 9 cases & $\begin{array}{l}16-49 \\
\text { years }\end{array}$ & 3 deceased & $\begin{array}{l}\text { Wolff-Parkinson-White syndrome, conduction system disease, and/or } \\
\text { hypertrophic cardiomyopathy [15] }\end{array}$ & G100S missense mutation ${ }^{a}$ \\
\hline Laforet et al. (2006) & 1 case & 38 years & $\begin{array}{l}\text { Not } \\
\text { deceased }\end{array}$ & $\begin{array}{l}\text { Sinusal bradycardia, high degree of ventricular block, } \\
\text { hypertrophic cardiomyopathy [18] }\end{array}$ & S548P missense mutation \\
\hline Murphy et al. (2005) & 44 cases & $\begin{array}{l}9-55 \\
\text { years }\end{array}$ & $\begin{array}{l}4 \text { disease } \\
\text { related } \\
\text { deaths }\end{array}$ & $\begin{array}{l}\text { Palpitation, dyspnea, chest pain, syncope, left ventricular } \\
\text { hypertrophy, ventricular pre-excitation (Wolff-Parkinson-White } \\
\text { Syndrome), myalgia, proximal myopathy, epilepsy [6] }\end{array}$ & $\begin{array}{l}\text { N488I mutation, R302G missense } \\
\text { mutation }\end{array}$ \\
\hline Burwinkel et al. (2005) & 1 case & 34 days & Deceased & $\begin{array}{l}\text { Fetal bradycardia, preventricular hypertrophy, cardiomegaly, } \\
\text { severe hypertrophic cardiomyopathy [19] }\end{array}$ & R531Q mutation \\
\hline Buhrer et al. (2003) & 1 case & 21 days & Deceased & $\begin{array}{l}\text { Bradycardia, atrial and biventricular hypertrophy, pericardial } \\
\text { effusion [20] }\end{array}$ & R531Q mutation \\
\hline Arad et al. (2002) & 6 cases & $\begin{array}{l}19-55 \\
\text { years }\end{array}$ & Unknown & $\begin{array}{l}\text { Cardiac hypertrophy, ventricular pre-excitation (Wolff-Parkinson-White } \\
\text { Syndrome), progressive dysfunction of the conduction system [8] }\end{array}$ & $\begin{array}{l}\text { R302G mutation, T400N } \\
\text { mutation, N488I missense } \\
\text { mutation }\end{array}$ \\
\hline Gollob et al. (2001) & 4 cases & $\begin{array}{l}8-41 \\
\text { years }\end{array}$ & Unknown & $\begin{array}{l}\text { Wolff-Parkinson-White Syndrome, ventricular preexcitation, early } \\
\text { onset of atrial fibrillation and conduction disease [21] }\end{array}$ & R531G missense mutation \\
\hline Regalado et al. (1999) & 2 cases & $\begin{array}{l}\text { Birth-2 } \\
\text { months }\end{array}$ & Deceased & Cardiomegaly, bradycardia, cardiorespiratory problems [22] & R531Q mutation \\
\hline Zhang et al. (2014) & $\begin{array}{l}\text { Zebrafish } \\
\text { cases }\end{array}$ & - & - & Thicker heart wall with increased glycogen storage [23] & G100S missense mutation ${ }^{a}$ \\
\hline
\end{tabular}

a Denotes same mutation as patient in case report (G100S).

with PRKAG2, epilepsy and muscle symptoms have been reported 7 of 40 patients (15\%) with a N4881 mutation in PRKAG2 had myalgia/myopathy Four patients from this cohort of 45 also had epilepsy (generalized tonic-clonic seizures), poorly controlled with medications, including 3 who also had myalgia [6]. The present report serves to add to the phenotypic spectrum of PRKAG2 as well as highlight the importance of confirming a diagnosis of Pompe disease by more than one method. The blood based assay to diagnose Pompe disease should be performed in a lab with experience because if not done correctly, the test can result in an incorrect initial diagnosis, as noted in this case. PRKAG2 syndrome should be considered in the differential diagnosis of Pompe disease. We have seen two additional cases of PRKAG2 syndrome where the patients were initially clinically misdiagnosed with Pompe disease due to significant hypertrophic cardiomyopathy at presentation in one case and muscle weakness in the other (PSK personal communication).

In the past, the diagnosis of Pompe disease was confirmed using GAA enzyme measurements in cultured fibroblasts or muscle cells. Enzyme measurement using acarbose, an inhibitor of alpha-glucosidase, can greatly improve the sensitivity and specificity of Pompe disease diagnosis in blood [16] and has now been adapted in many labs as a rapid way to diagnose Pompe disease; however, without the addition of acarbose, there can be false positive results. Thus, the test needs to be done in labs with experience and expertise. We stress the importance of broadening the diagnostic measures to include additional tests outside of enzyme testing in dried blood spots (DBS) such as gene sequencing and measurement of GAA activity in other tissue such as skin and muscle prior to initiation of ERT [17].

Among the GSDs, Pompe disease is the only exception with glycogen accumulation in lysosomes (lysosomal GSD) whereas all others have glycogen storage in the cytoplasm (cytoplasmic GSD). Furthermore, the use of ERT with alglucosidase alfa depends upon the mannose 6phosphate receptor mediated enzyme uptake into lysosomes, which has been effective in reducing lysosomal glycogen storage in Pompe disease. Our group has demonstrated that ERT significantly reduced glycogen levels in the cultured primary myoblasts from skeletal muscle biopsies of patients with GSD IIIa, a cytoplasmic GSD caused by the deficiency of glycogen debranching enzyme that leads to accumulation of abnormally structured cytoplasmic glycogen in liver and muscle [11]. We speculated that administration of recombinant human acid alfa glucosidase enhanced lysosomal glycogen depletion, facilitated glycogen transport from the cytoplasm into lysosomes, and ultimately reduced cytoplasmic glycogen accumulation in the GSD III patient cells. As evidenced by the outcomes of this case depicting significant musculoskeletal improvements with alglucosidase alfa, a subsequent decline when ERT was withdrawn, and then improvement following reinitiation of ERT, there seem to be implications of the effectiveness of alglucosidase alfa therapy for PRKAG2 syndrome. It is possible that physical therapy and endurance exercise could be adding to the improvement in strength in this patient. However, this patient continued PT throughout his clinical course, even when ERT was discontinued with no clinical impact. It is also possible that being a carrier for Pompe disease which resulted in a decrease in residual endogenous GAA activity could have resulted in an even greater clinical benefit from the administration of recombinant human GAA (ERT) in this case. However, in the preclinical work with GSD III (cited above), even with normal GAA activity, a benefit in cytoplasmic glycogen clearance was noted. Thus, we would expect the benefit, even if the patient was not a carrier for Pompe disease.

More clinical studies are required to confirm the treatment effect of ERT with recombinant human GAA in PRKAG2 and other cytoplasmic GSDs. This report supports our previous findings of the use of alglucosidase alfa as a novel therapy for cytoplasmic GSDs [10] like PRKAG2 syndrome.

PSK and BS are inventors on technology used in the study, which technology has been patented and licensed to a company for commercial development. The developers and Duke University may benefit financially if that commercial development is successful.

\section{References}

[1] Y.T. Chen, D. Koeberl, Glycogen storage diseases, in: D. Valle, et al., (Eds.), Scriver's Online Metabolic \& Molecular Bases of Inherited Disease, McGraw-Hill, New York, 2009.

[2] C. Moffat, M.E. Harper, Metabolic functions of AMPK: aspects of structure and of natural mutations in the regulatory gamma subunits, IUBMB Life 62 (10) (2010) 739-745.

[3] B.B. Kahn, et al., AMP-activated protein kinase: ancient energy gauge provides clues to modern understanding of metabolism, Cell Metab. 1 (1) (2005) 15-25.

[4] A. Marette, The AMPK signaling cascade in metabolic regulation: view from the chair, Int. J. Obes. 32 (4) (2008) S3-S6.

[5] M. Arad, et al., Glycogen storage diseases presenting as hypertrophic cardiomyopathy, N. Engl. J. Med. 352 (4) (2005) 362-372.

[6] R.T. Murphy, et al., Adenosine monophosphate-activated protein kinase disease mimicks hypertrophic cardiomyopathy and Wolff-Parkinson-White syndrome: natural history, J. Am. Coll. Cardiol. 45 (6) (2005) 922-930. 
[7] A.G. Porto, et al., Clinical spectrum of PRKAG2 syndrome, Circ. Arrhythm. Electrophysiol. 9 (1) (2016), e003121.

[8] M. Arad, et al., Constitutively active AMP kinase mutations cause glycogen storage disease mimicking hypertrophic cardiomyopathy, J. Clin. Invest. 109 (3) (2002) 357-362.

[9] R.W. Hunter, et al., Molecular mechanism by which AMP-activated protein kinase activation promotes glycogen accumulation in muscle, Diabetes 60 (3) (2011) 766-774.

[10] P.S. Kishnani, et al., A retrospective, multinational, multicenter study on the natural history of infantile-onset Pompe disease, J. Pediatr. 148 (5) (2006) 671-676.

[11] B. Sun, et al., Alglucosidase alfa enzyme replacement therapy as a therapeutic approach for glycogen storage disease type III, Mol. Genet. Metab. 108 (2) (2013) $145-147$.

[12] S.E. Henderson, D.A. Sugden, Movement Assessment Battery for Children, Psychological Corporation, London, 1992.

[13] Y.P. Wuang, J.H. Su, C.Y. Su, Reliability and responsiveness of the movement assessment battery for children-second edition test in children with developmental coordination disorder, Dev. Med. Child Neurol. 54 (2) (2012) 160-165.

[14] L.J. Miller, Miller function and participation scales, Pearson Education, 2006.

[15] B.L. Zhang, et al., Identification and functional analysis of a novel PRKAG2 mutation responsible for Chinese PRKAG2 cardiac syndrome reveal an important role of nonCBS domains in regulating the AMPK pathway, J. Cardiol. 62 (4) (2013) 241-248.

[16] N.A. Chamoles, et al., Glycogen storage disease type II: enzymatic screening in dried blood spots on filter paper, Clin. Chim. Acta 347 (1-2) (2004) 97-102.
[17] Pompe Disease Diagnostic Working, G., et al., Methods for a prompt and reliable laboratory diagnosis of Pompe disease: report from an international consensus meeting, Mol. Genet. Metab. 93 (3) (2008) 275-281.

[18] P. Laforet, et al., A new mutation in PRKAG2 gene causing hypertrophic cardiomyopathy with conduction system disease and muscular glycogenosis, Neuromuscul. Disord. 16 (3) (2006) 178-182.

[19] B. Burwinkel, et al., Fatal congenital heart glycogenosis caused by a recurrent activating R5310 mutation in the gamma 2-subunit of AMP-activated protein kinase (PRKAG2), not by phosphorylase kinase deficiency, Am. J. Hum. Genet. 76 (6) (2005) 1034-1049.

[20] C. Buhrer, et al., Fetal bradycardia at 28 weeks of gestation associated with cardiac glycogen phosphorylase b kinase deficiency, Acta Paediatr. 92 (11) (2003) 1352-1353.

[21] M.H. Gollob, et al., Novel PRKAG2 mutation responsible for the genetic syndrome of ventricular preexcitation and conduction system disease with childhood onset and absence of cardiac hypertrophy, Circulation 104 (25) (2001) 3030-3033.

[22] J.J. Regalado, M.M. Rodriguez, P.L. Ferrer, Infantile hypertrophic cardiomyopathy of glycogenosis type IX: isolated cardiac phosphorylase kinase deficiency, Pediatr. Cardiol. 20 (4) (1999) 304-307.

[23] B.L. Zhang, et al., Overexpression of G100S mutation in PRKAG2 causes WolffParkinson-White syndrome in zebrafish, Clin. Genet. 86 (3) (2014) 287-291. 